\title{
The MRC trial of assessment and management of older people in the community: objectives, design and interventions [ISRCTN23494848]
}

\author{
AE Fletcher*1, DA Jones ${ }^{2}$, CJ Bulpitt ${ }^{3}$ and AJ Tulloch ${ }^{4}$
}

Address: ${ }^{1}$ Centre for Ageing and Public Health, London School of Hygiene and Tropical Medicine, Keppel St, London WC1E 7HT, UK, ${ }^{2}$ University Department of Geriatric Medicine, Llandough Hospital, Penlan Rd, Penarth, Cardiff, CF64 2XX, UK, ${ }^{3}$ Section of Care of the Elderly, Faculty of Medicine, Imperial College, Hammersmith Campus, Du Cane Road, London W12 ONN, UK and ${ }^{4}$ Unit of Health Care Epidemiology, Institute of Health Sciences, University of Oxford, OX3 7LF, UK

E-mail: Fletcher AE* - Astrid.Fletcher@Lshtm.ac.uk; Jones DA - dee.jones@virgin.net; Bulpitt CJ - c.bulpitt@ic.ac.uk; Tulloch AJ - aj.tulloch@btinternet.com

*Corresponding author

Published: 25 October 2002

BMC Health Services Research 2002, 2:21

This article is available from: http://www.biomedcentral.com/I472-6963/2/2 I

(C) $2002 \mathrm{AE}$ et al; licensee BioMed Central Ltd. This article is published in Open Access: verbatim copying and redistribution of this article are permitted in all media for any purpose, provided this notice is preserved along with the article's original URL.
Received: 20 June 2002
Accepted: 25 October 2002

\begin{abstract}
Background: The benefit of regular multidimensional assessment of older people remains controversial. The majority of trials have been too small to produce adequate evidence to inform policy. Despite the lack of a firm evidence base, UK primary care practitioners (general practitioners) are required to offer an annual health check to patients aged 75 years and over.

Design: Cluster-randomised factorial trial in primary care comparing a package of assessments (i) universal versus targeted assessment and (ii) management by the primary care team (PC) or a multidisciplinary geriatric assessment team (GM). The unit of randomization is the general practice.

Methods: Older people aged 75 and over eligible for the over 75 s health check and excluding those in nursing homes or terminally ill were invited to participate. All participants receive a brief assessment covering all areas of the over $75 \mathrm{~s}$ check. In the universal arm all participants also receive a detailed health and social assessment by a study nurse while in the targeted arm only participants with a pre-determined number and range of problems at the brief assessment go on to have the detailed assessment. The study nurse follows a standard protocol based on results and responses in the detailed assessment to make referrals to (i) the randomised management team (PC or GM) (ii) other medical services, health care workers or agencies (iii) emergency referrals to the GP. The main outcomes are mortality, hospital and institutional admissions and quality of life. 106 practices and 33,000 older people have been recruited to the trial.
\end{abstract}

\section{Background}

Indications for possible benefit from regular assessment of elderly people came from early studies several decades ago in the UK which found high levels of undetected problems in elderly people [1-3] and highlighted the need for a systematic approach to problem detection. Around the same era, an approach emerged which emphasized function and disability in the medical care of elderly people and questionnaires were developed to assist in the assessment of a range of physical, mental and 
social dimensions [4]. Doubts about the feasibility of routine assessment of elderly people [5] led to the notion of two stage targeted screening to identify those at greatest risk or greatest need [6-8]. In the 1980s results from three randomised controlled trials, which examined the benefit of socio-medical assessment, were published [9-12]. Two of these trials took place in the UK $[9,10]$ in the setting of general practice and one in Denmark among elderly people living in the community $[11,12]$. These trials suggested some possible benefits on mortality and hospital admissions (mainly the Danish trial) but none of the trials provided convincing evidence for regular assessment of elderly people.

Despite these equivocal results, the Department of Health in 1990 introduced a contract of service for general practitioners, which required them to offer an annual assessment to patients aged 75 years, and over [13]. Although the contract specified the broad areas for assessment (Table 1), the method, level and nature of assessment were not defined. Different models of initial assessment have been used (e.g. postal, lay person and nurse) and targeted screening has been recommended [14] but none of these strategies have been rigorously evaluated and compared. Models of subsequent management of problems identified through the assessment process also require evaluation. In studies conducted mainly in North America, multidisciplinary teams in the hospital setting (usually geriatrician, nurse, therapist and social worker) appear to offer advantages in terms of survival, functional status and use of hospital services [15], but the costs and benefits of the multidisciplinary team as a integrated component of multidimensional assessment, when compared with the usual model of primary care, have yet to be established.

The principal objective of the MRC trial of assessment and management of older people in the community is to evaluate a package of multidimensional assessment and management of older people in the context of the 1990 contract of service. The two main components of the package are the method of assessment - Universal or Targetedand the method of clinical management - multidisciplinary geriatric team (GM) or usual primary care (PC). A secondary objective is to compare different methods of administering a brief screening questionnaire (postal, lay or nurse) These three methods were chosen because they that had been advocated as part of an assessment process (14) (with obvious differences in cost implications) but no formal evaluation of their performance in a single trial has been carried out. We were interested in establishing whether response rates, levels of missing information and, for certain health conditions, sensitivity and specificity compared to measurements at the detailed assessment, varied for the three methods.
Table I: Department of Health Contract of Service with General Practitioners 1990

Annual invitation to each patient aged 75 and over to participate in consultation

Assessment should include where appropriate

- sensory function

- mobility

- mental condition

- physical condition including continence

- use of medicines

- social environment

\section{Design}

The study is a cluster-randomised trial with a $2 \times 2$ factorial design i.e. practices are randomised to: Universal assessment (plus randomised to GM or PC) or Targeted (plus randomised to GM or PC) (Figure 1). In addition to the main randomisations, practices are also randomised to one of the three methods of administering a brief questionnaire, which are balanced across the main randomised groups. All randomisation is at the level of the general practice.

\section{Description of procedures}

All practices administer a brief assessment questionnaire to participants in the study either by post, or by a layperson, or by a nurse. Following the brief assessment, practices in the Universal arm carry out a detailed examination by the study nurse in all patients (irrespective of their responses on the brief assessment) while those in the Targeted arm carry out a detailed assessment only in those patients who "trigger" on the brief assessment. The study nurse follows a standard protocol based on results and responses in the detailed assessment to make referrals to (i) the randomised team (PC or GM)(ii) other medical services, health care workers or agencies (iii) emergency referrals to the GP.

\section{Methods}

\section{Methods of assessment}

The brief assessment questionnaire

The brief assessment questionnaire, developed in conjunction with Wallace and Williams (who have published the binary response version of the questionnaire) [16] covers all the areas specified in the GP contract: social environment, activities of daily living, sensory problems, mobility, physical symptoms including continence, mental condition, use of medication. We chose to use a graded response scale based on the results of pilot studies comparing graded with binary responses, which showed that binary responses inflated positive responses and that patients had difficulty making choices on a binary scale. The areas and the corresponding questions and scale are 

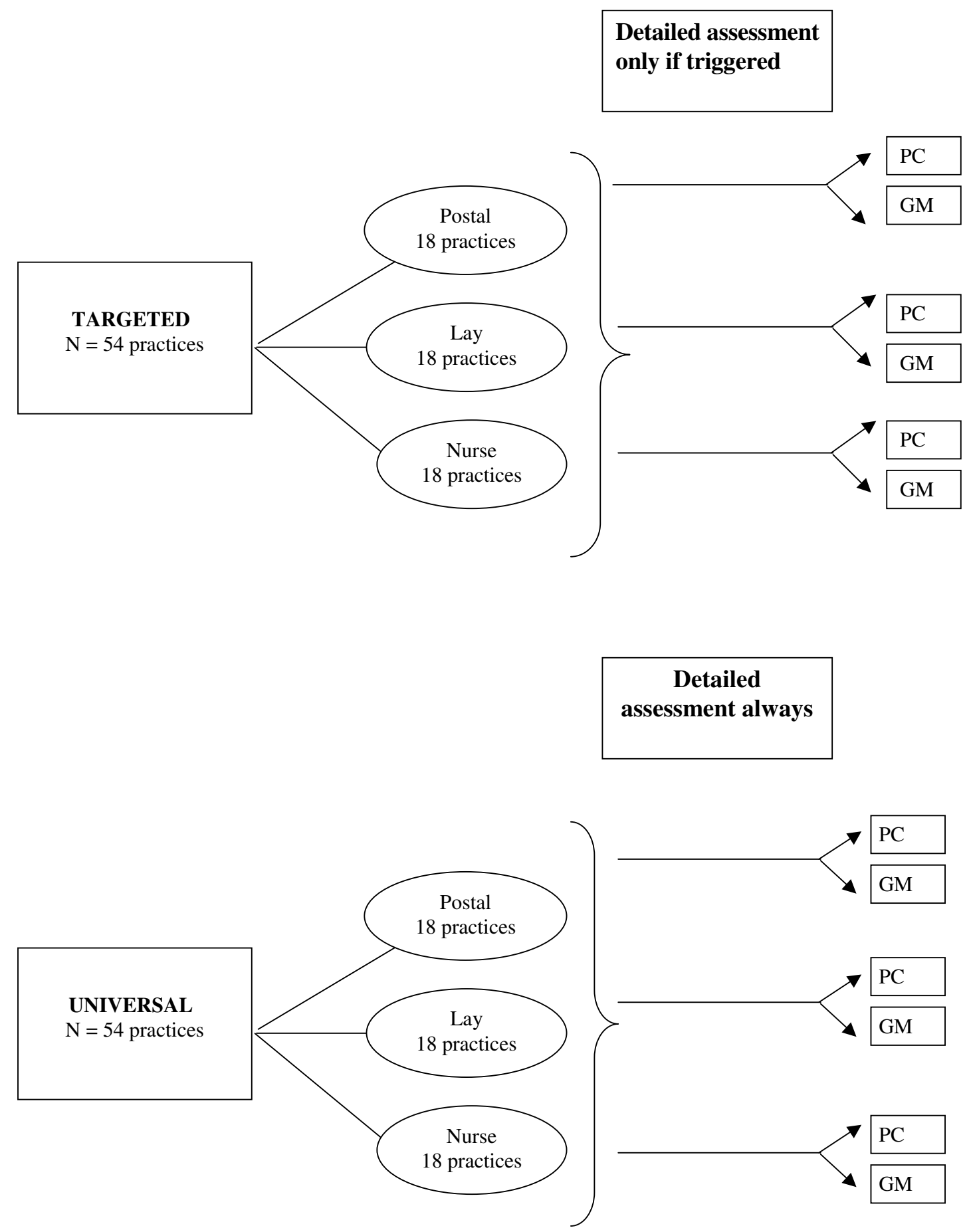

Figure I

Design of MRC trial of assessment and management of older people in the community 
Table 2: The brief assessment questionnaire areas, specified in the GP contract and corresponding questions

\begin{tabular}{|c|c|c|}
\hline Area & Questions & Response scale and trigger \\
\hline \multirow[t]{11}{*}{ Social Environment } & Social support & \\
\hline & $\begin{array}{l}\text { Living circumstances Carer for someone else at } \\
\text { home Someone to call on for help Frequency of } \\
\text { social contacts }\end{array}$ & $\begin{array}{l}\text { Live alone or is carer and nobody to call on for } \\
\text { help or rarely sees relatives/friends }\end{array}$ \\
\hline & Self care & \\
\hline & Wash all over & Unable and no help \\
\hline & Get dressed & Unable and no help \\
\hline & Cut toe nails & Unable and no help \\
\hline & Cook hot meal & Unable and no help \\
\hline & Do light housework or simple repairs & Unable and no help \\
\hline & Financial problems & \\
\hline & Difficulty keeping home warm & Often or always \\
\hline & Problems in making ends meet & Always \\
\hline \multirow[t]{2}{*}{ Sensory impairment } & Difficulty hearing & A lot \\
\hline & Difficulty seeing newsprint & A lot \\
\hline \multirow[t]{4}{*}{ Mental condition } & Feeling sad, depressed or miserable & Often or always \\
\hline & Problems with everyday memory & Always \\
\hline & Difficulty managing finances & Always \\
\hline & Problems remembering medication & Often or always \\
\hline \multirow[t]{6}{*}{ Physical condition } & Vomited blood & Yes' \\
\hline & Coughed up blood & Yes' \\
\hline & Severe shortness of breath sitting & Yes \\
\hline & Severe swollen legs & Yes \\
\hline & Unexpected weight loss & Yes' \\
\hline & Falls in last six months & $>4^{1}$ \\
\hline \multirow[t]{2}{*}{ Incontinence } & Urinary & Once a week or more often \\
\hline & Faecal & Once a week or more often \\
\hline Use of medicines & Number of prescribed medicines & $>7$ \\
\hline \multirow[t]{3}{*}{ Mobility } & Walk 50 yards & Unable and no help \\
\hline & Go up and down stairs and steps & Unable and no help \\
\hline & Do shopping & Unable and no help \\
\hline Lifestyle (not part of GP contract) & $\begin{array}{l}\text { Use of alcohol in previous week Current smoker } \\
\text { (amount daily) Physical activity }\end{array}$ & Not included as trigger \\
\hline For nurse/lay only & & $\begin{array}{l}\text { Any other condition/problem warranting a detailed } \\
\text { assessment }\end{array}$ \\
\hline
\end{tabular}

I Potentially serious symptom triggering detailed assessment

shown in Table 2. Additional questions on alcohol consumption, cigarette smoking and physical activity have been included for epidemiological purposes. Criteria for triggering to the detailed assessment are 3 or more problems identified from the brief assessment or any one of 4 "serious" symptoms. According to the randomisation, patients are interviewed either by a lay person (usually a member of the practice staff) or the study nurse or the questionnaire is mailed. Patients are invited to attend the surgery for the interview but are given the option of the interview being carried out at home. Identical questions are asked in each version of the questionnaire but for the postal questionnaire the scoring of the triggers is carried out by the study nurse. The postal questionnaire was printed in large font to permit easy reading.
The detailed assessment

Patients are invited to attend the surgery for the detailed assessment but are given the option of the assessment being carried out at home. The detailed assessment covers the same areas as specified above but in greater depth for example, whispered voice test for hearing [17], Glasgow Acuity Cards for vision [18], Mini Mental State Examination for cognitive impairment [19], the Geriatric Depression Scale [20]. Additional questions include more detailed assessment of symptoms (e.g. Rose chest pain questionnaire for possible angina [21], respiratory problems, urinary and faecal incontinence, examination of legs and feet and a modified version of a checklist for possible drug interactions [22]. Additional biological measurements include blood pressure, heart rate, and dipstick for 
Table 3: Criteria for referral to primary care team (PC) or geriatric evaluation and management team (GM) I

\begin{tabular}{|c|c|}
\hline Clinical domain & Referral Criteria \\
\hline Bradycardia & Heart rate $<50$ \\
\hline Tachycardia & Heart rate $>=110$ \\
\hline Abnormal ECG & ECG carried out for irregular pulse \\
\hline \multirow[t]{3}{*}{ Hypertension } & Aged less than 80 years average repeat sitting systolic \\
\hline & $>=180 \mathrm{mmHg}$ or sitting diastolic \\
\hline & $\begin{array}{l}>=100 \mathrm{mmHg} \text {. To refer for either, } \\
\text { standing systolic must be }>=140 \mathrm{mmHg}\end{array}$ \\
\hline Untreated Angina & Positive on Rose chest pain questionnaire \\
\hline Severe leg oedema & Swelling of legs up to knees on getting up in the morning \\
\hline Severe shortness of breath & Short of breath on talking \\
\hline Weight loss & Recent unexplained weight loss of more than half a stone \\
\hline Depression & GDS score $>7$ and no treatment or more than 6 months on present treatment \\
\hline History of recent falls & $>4$ falls in previous 6 months \\
\hline Infected MSU & MSU tested when patient reports urinary incontinence or has proteinuria or haematuria on dipstix \\
\hline Other urinary problems (men) & Difficulty in micturition Nocturia more than twice nightly \\
\hline Faecal incontinence & Soiling 3 or more times a week \\
\hline Faecal occult blood & Blood in motions and stool specimen is positive for blood \\
\hline Change in bowel habits & Constipation Diarrhoea \\
\hline Haematemesis & Recent history of vomiting blood \\
\hline Haemoptysis & Recent history of coughing up blood \\
\hline Dysphagia & Difficulties swallowing \\
\hline Glycosuria & Positive for glucose on dipstix \\
\hline Abnormal biochemistry & $\begin{array}{l}\text { Outside normal ranges for haemoglobin, white cell count, platelets, TSH, glucose, sodium, potassium, } \\
\text { urea, creatinine, albumin, calcium, bilirubin, alkaline phosphatase, aspartase-transaminase }\end{array}$ \\
\hline Potential drug interactions & Modified version of the Stockley checklist \\
\hline $\begin{array}{l}\text { Any other serious condition warranting } \\
\text { further investigation }\end{array}$ & Nurse judgement \\
\hline
\end{tabular}

I Excludes details on criteria for emergency referrals

blood, protein, and urine. In all patients a blood sample is taken for a full biochemical screen. Additional laboratory investigations are triggered by question responses or abnormal results e.g. faecal occult testing for a positive response to a question on blood in the stools, an MSU for a positive dipstick result for protein or blood. Patients are also assessed for social problems such as financial difficulties, social isolation. The choice of conditions to screen for was based on a review of the available literature [23]. The study nurse follows a protocol, based on results and responses in the detailed assessment, to make referrals to (i) the clinical teams (PC or GM) (ii) other medical services, health care workers or agencies (iii) emergency referrals to the GP. Referrals to PC/GM are for serious clinical problems, such as depression, frequent falls, abnormal biochemical results, severe breathlessness, and severe leg oedema. A full list is given in Table 3 . The conditions for referral to the teams were based on the results of a survey of 90 general practitioners from the MRC General Practice Research Framework (MRC GPRF) and 52 geriatricians, who had indicated an interest in taking part in the study. Other referrals are to specialities or professions for a vari- ety of problems, such as ophthalmology for visual impairment (not due to refractive errors), audiology, continence advisors, community psychiatric nurse, social services (Table 4). For certain problems, patients are advised to seek a consultation e.g. with an optician for refractive error (VA<6/18 corrected with pinhole), or to a dentist for problems with mastication.

\section{Management teams}

The teams follow their "usual" practice and there was no attempt to impose a formal protocol. Information is collected on investigations, diagnoses, services and treatments that result from the referral and any further referrals.

\section{Outcome measures}

The principal outcome measures for hypothesis testing are mortality, hospital and institutional admissions and quality of life. Mortality follow-up is achieved by registering all eligible patients with ONS for notification of death, date and cause of death. Hospital admissions are collected for each participant for a 2-year period from the time of the 
Table 4: Detailed assessment: referrals to other health professionals/agencies

\begin{tabular}{|c|c|c|}
\hline Referral to & Problem & Referral criteria \\
\hline Audiology & Hearing impairment & Fail whispered voice test and no wax \\
\hline Ophthalmologist & Vision impairment & $V A<6 / 18$ in either eye not corrected by pinhole \\
\hline Memory Clinic /CPN & Moderate to severe cognitive impairment & $\begin{array}{l}\text { Mini Mental state }<17 \text { or }<12 \text { if language section could not be } \\
\text { completed }\end{array}$ \\
\hline Continence advisor/community nurse & Urinary incontinence & more than once a week and MSU not infected. \\
\hline Community nurse & Leg/foot ulcers/ bed sores & Present on nurse examination \\
\hline Chiropodist & Foot problems & $\begin{array}{l}\text { Corns, bunions, ingrowing or long toe nails or other treatable } \\
\text { foot problem on examination }\end{array}$ \\
\hline \multirow[t]{4}{*}{ Social services } & Self-care & $\begin{array}{l}\text { No help available for difficulties with dressing, cooking hot } \\
\text { meal, carrying out light housework, washing and shopping }\end{array}$ \\
\hline & Social isolation & Lack of close confident or someone to call on for help \\
\hline & Financial problems & Difficulty in making end meet Difficulty in paying bills \\
\hline & Housing problems & Difficulty in keeping home warm No indoor toilet \\
\hline
\end{tabular}

brief assessment using information from hospital discharge letters in the patients' GP records. Information collected includes specialty, dates of admission and discharge, diagnoses, specialty of consultant. The discharge letter is considered to be a reliable source since this is the routine method of providing information to general practitioners from hospital services. Institutional admissions are collected on an ongoing basis for each patient from the date of the baseline assessment. Quality of Life interviews are carried out in the patient's own homes by fieldworkers who are independent of the practice. The interviews take place (i) at baseline and prior to the assessment (ii) 18 months following the baseline interview (iii) 36 months following the baseline. The Quality of Life core interview schedule includes four dimensions from the Sickness Impact Profile (SIP) (mobility, self-care, homemanagement, social interaction) [24], and the Philadelphia Geriatric Morale Scale (PGMS), [25], a 17-item measure of morale specifically developed for use with older people.

We used slightly different follow-up periods for outcome collection since we would expect any effect on hospital admissions to be seen at an earlier rather than later stage whereas mortality effect might show some lag. Quality of life is measured both at 18 months and at 3 years, which covers the same period as both the hospital admissions and the mortality.

\section{Use of services}

Information on use of services is being collected for the economic analysis. Use of public sector services (health and social services) is collected through two sources (i) in the quality of life questionnaire at baseline, 18 and 36 months) (ii) through a postal questionnaire mailed to randomly selected cross sectional samples of patients at 6 monthly intervals across the follow-up period. This permits us to have information both from a longitudinal sample over the 3 years of the trial from participants in the quality of life sample while the cross sectional random sample provides a more representative sample across the follow-up period since it includes those those who may die or leave the practice.

\section{Trial hypotheses and sample size}

Sample size methods

Sample size calculations for mortality and hospital admissions were calculated from a program provided by Martin Shipley at University College London, Department of Epidemiology, using methods for cluster randomisation described in Shipley et al [25] and under the assumption that the rates follow a Poisson distribution. The program uses the formula

$$
C_{\boldsymbol{b}}=\frac{|\Delta| m}{\left(\frac{(2 \boldsymbol{l}+\Delta)}{n_{H}}+2 \boldsymbol{s}^{2}(1-\kappa)\right)^{1 / 2}(m+2)^{1 / 2}}-C_{\boldsymbol{a} / 2}
$$

where study power is given by $1-\beta, C_{\beta}$ is the critical value of the normal distribution in the upper tail of the standard normal curve, $\mathrm{C}_{\alpha / 2}$ is the critical value of the normal distribution for a two sided value of alpha (in our case taken to be alpha $=0.01), \lambda$ is the average value of the underlying event rate, $\Delta$ is the difference to be detected between the event rate in the main randomised groups of the trial (universal minus targeted or GM minus PC), $2 \sigma^{2}(1-\kappa)$ is the variance of the underlying event rates, $\mathrm{n}_{\mathrm{H}}$ is the har- 
monic mean of all $2 \mathrm{~m}$ sample sizes and $2 \mathrm{~m}$ is the number of clusters. We took the conservative assumption that the level of matching of the practices would be minimal or essentially zero although we did attempt to balance the practices by Jarman score and SMR by stratified randomization across the joint tertiles of SMR and Jarman. Expected mortality rates were based on ONS statistics for England and Wales [26] with an adjustment downwards of 0.7 of the background mortality rate on the basis that the trial population would have lower rates (healthy participant effect). Rates of hospital admission [27] were adjusted down on the basis of an expected "healthy" participant effect and additionally on the basis that hospital admissions might be under reported and that annual rates do not distinguish between persons and admissions. For hospital admission rates we therefore assumed that the rates might be a half of those published. Thus in both calculations we used conservative estimates of the rates. Sample sizes for institutional admissions could not be calculated as there are no national data on rates of institutional admission. The sample size calculations assumed an average of 500 eligible patients per cluster followed for an average of 3 years.

Sample size estimates for the quality of life measures used the formula given by Hsieh [28] based on the $\mathrm{Z}$ approximation,

$\mathrm{N}=8\left(\mathrm{~S}_{\mathrm{b}}{ }^{2}+\mathrm{S}_{\mathrm{w}}{ }^{2} / \mathrm{m}\right)\left(\mathrm{z}_{\alpha / 2}+\mathrm{z}_{\beta}\right)^{2} / \mathrm{d}^{2}$

where $\mathrm{N}$ is the total number of clusters, $\mathrm{S}_{\mathrm{b}}{ }^{2}$ is the between cluster component of variance, $\mathrm{S}_{\mathrm{w}}{ }^{2}$ is the within cluster component of variance, $\mathrm{m}$ is the number of individuals within each cluster, and $z_{\alpha / 2}$ and $z_{\beta / 2}$ are the critical points of the normal distribution for alpha of 0.01 and 1$\beta$ of 0.9 and $\mathrm{d}$ is the difference between randomised groups in the means of qol scores. Estimates of $\mathrm{S}_{\mathrm{w}}{ }^{2}$ and $\mathrm{d}^{2}$ were based on SIP scores from pilot studies of 52 older people attending geriatric out patient clinics and POMS scores from 115 patients on the care of the elderly wards at the Hammersmith Hospital. We expected $75 \%$ of baseline responders to provide quality of life data at the 3 year end point $(\mathrm{m}=350)$. Since no data were available for $\mathrm{S}_{\mathrm{b}}{ }^{2}$ (which is expected to be considerably smaller than $S_{w}{ }^{2}$ ) we used varying estimates of the intraclass correlation coefficient $\left(\mathrm{S}_{\mathrm{b}}^{2} / \mathrm{S}_{\mathrm{b}}^{2}+\mathrm{S}_{\mathrm{w}}^{2}\right)(0.01,0.02,0.04)$.

\section{Estimates of sample size}

108 practices (with an average of 500 patients aged 75 years and over) are required to detect differences between Stage 1 assessment methods (targeted versus universal) of: at least $15 \%$ in mortality assuming a background mortality rate of approximately 60/1000 person years over an average follow up period of 3 years; at least $10 \%$ in 2 year hospital admissions assuming a background first admis- sion rate of $150 / 1000$ person years. This sample size is sufficient to detect differences between the two management strategies (GM and PC) of at least 22\% in mortality and at least $15 \%$ in hospital admissions. Smaller numbers of practices are required to detect differences in quality of life. A random sample of 24 practices is adequate to detect differences between Stage 1 assessment methods of at least 15\% in PGMS and SIP and between GM and PC of at least 25\% in PGMS and SIP. The size of the effects to be tested were based on previous trials and a realistic expectation of what might be achievable and of public health importance.

\section{Trial population}

The trial is being conducted in practices recruited through the MRC GP Research Framework with list sizes of between 200 and 700 patients aged 75 years and over and selected to be representative of the joint tertiles of Jarman and Standard Mortality Ratios (SMRs) in UK practices. To be eligible for randomisation in the trial, recruited practices first had to obtain the agreement of the local geriatrician to participate (in order to ensure no selection bias of geriatricians in practices subsequently randomised to GM or PC). Eligible patients were aged 75 years and over in the year the practice undertook the assessments, excluding anyone in long-term care or with terminal illness. Patients in residential or sheltered accommodation were included.

\section{Method of allocation to groups}

Allocation to groups was by a computer generated randomisation list, stratified by Jarman and SMR tertile as practices were recruited to the trial.

\section{Informed consent}

The over $75 \mathrm{~s}$ check is a contractual obligation of general practitioners. The letter of invitation to the study was done in the context of the letter of invitation to the annual check. The letter of invitation informed patients that the check could be done as part of a research study with a brief description of the intervention (this varied according to the practice randomisation). Patients were given the opportunity to have the check carried out in the usual way and reassured that non-participation in the study would not affect their usual care.

\section{Ethical approval}

Local Research Ethics Committee approvals were obtained for all the practices participating in the trial.

\section{Analysis}

The principal analysis is of the components of the package of interventions and therefore all analyses will take account of the other main randomisation. The results will be presented for (i) universal versus targettted (ii) GM versus PC. In order to ensure that the method of administering 
the brief questionnaire does not influence the main randomisations, the analyses will also be adjusted for the 'nurse $v$ s. lay $v s$. postal' randomised intervention. The primary population for analysis is the "Intention to Treat" population (i.e. all those eligible for the trial and invited to take part irrespective of participation). Pre-specified covariates are age, sex and (at practice level) SMR and Jarman score. There will be no sub-group analyses in the primary analysis. All analyses will take account of cluster randomisation which has the effect of increasing the standard errors of the estimates and therefore it is unlikely that spurious significant effects will be detected. Secondary analyses will be conducted as above on the "per protocol population"

\section{Economic analysis}

The primary objective of the economic analysis is to determine the relative costs and costs per life year gained of the different components of the assessment and management packages. Cost analysis will compare the direct health and social care costs of assessments and the longer term costs of care and treatment following the interventions (use of services, hospital admissions, and instiututional admissions). Service use data over the follow-up period of the trial will be obtained from longitudinal samples (as part of the quality of life questionnaire) and from the repeated cross sectional samples.

\section{Progress on trial}

109 practices were randomised and study staff trained in the study protocol; three practices withdrew before carrying out any of the study interventions (one of these was also randomised to the quality of life collection). It was too late to replace these practices so the final achieved target is 3 practices short. 106 practices and 33,000 patients are participating in the trial. The practices are equally distributed over the Universal $(n=53)$ or Targeted $(n=53)$ arms and of the PC $(n=53)$ or GM $(n=53)$ arms of the trial.

\section{Discussion}

This trial is, by far, the largest and most comprehensive study internationally to examine the benefits of multidimensional assessment of older people in the community. Although a number of trials have been published since the introduction of the 1990 contract, including three from the UK, they have, in common with previous trials, been too small to produce results of sufficient precision and certainty to inform policy decisions [29]. Studies conducted in the US are difficult to translate to the UK health care setting. Previous trials have also suffered from a number of other methodological problems: individual patient randomisation in the same healthcare setting (e.g. general practice) which may lead to contamination of randomised groups and dilution of effect; lack of a clearly de- fined protocol for intervention and referral; and (in the US studies) low participation rates and over-representation of fit older people. One systematic review and three meta analyses of these trials have been published [30-33], but have come to different conclusions concerning benefits on major outcomes, such as mortality and hospital admissions. Our trial avoids some of these design problems by employing: randomisation at the general practice level, which reduces the possibility of contamination; clearly defined protocols for the intervention and referrals; minimal exclusion criteria (terminally ill or in institutional care); high power to test hypotheses on major outcomes. These outcomes were chosen because a number of trials had suggested possible benefits on mortality, institutional (or nursing home) and hospital admissions. Individual studies and meta analyses have also looked at outcomes such as functional decline or physical morbidity - we used comparable domains of health related quality of life as an outome to capture these aspects and also included morale and social interaction as other relevant outcomes. Our trial compares different strategies of multidimensional geriatric assessment. These different levels are analogous to the different intensities of screening described by Stuck and colleagues in their recent meta analysis (33), for example universal assessment with management by a specialist geriatric team in our trial is comparable to their category of "multidimensional geriatric assessment and follow-up" in the meta-analysis. Hence our results are applicable across differing health care contexts. Our trial interventions and referrals across a broad range of health care professionals and other agencies address disability and impairment as well as disease which we consider to be an appropriate balance rather than adopting either a purely medical or functional model of health at older ages.

There are a number of limitations of our trial. Cluster randomisation trials require substantially greater numbers than trials of individual randomisation, and are more susceptible to practice effects e.g. loss of all patients to follow-up if a practice withdraws. The large numbers also made certain aspects of data collection prohibitively expensive. For this reason we have not collected any detailed information on services and treatments already being received by trial participants ie prior to the trial interventions, although we will have some limited information on these if part of the criteria for referral. Also, funding did not permit collection on hospital admissions over a longer period. Nor were we able, for costs reasons, to repeat the intervention packages over several years or, to examine the benefits of annual assessment versus some other time period such a triennial assessment. A further limitation of our trial design was that it was not possible to have a control group (i.e. a group randomised to no intervention) because of the contractual obligation of general 
practitioners to offer an annual health check to the over $75 \mathrm{~s}$. It is extremely unfortunate that this policy was introduced (despite the lack of a firm evidence base) because it has meant that a properly controlled study cannot be conducted in the UK.

As with other trials, especially in health services research, there are issues of generalisability of the results to other relevant health care professionals (practice nurses, general practitioners and geriatricians) and to patients. The practices in the trial belong to the MRC General Practice Research Framework and, although the patients in those practices were representative of the wider patient population (in terms of deprivation and mortality), the general practitioners have a interest in being involved in research. However we have no reason to believe that the general practitioners or the nurses had any particular expertise in geriatric assessment or management. The geriatricians were recruited to the study by virtue of being the linked local geriatric service to the general practice and were not selected for any particular expertise or experience in research. Ensuring agreement of the local geriatricians to take part in principal, which was a criterion for randomisation of the practice (i.e. before knowledge of whether the practice was randomised to $\mathrm{PC}$ or GM), reduced the likelihood of selection bias of geriatricians. Nonetheless it will be important to describe the clinical management through the collection of process information so that, if the trial shows a benefit for a particular package of assessment and management, its components can be described.

The trial results are also of direct relevance to the "single assessment " recommended by the National Service Frameworks for Older people [34], in particular the decision on how and to whom the brief overview assessment should be targeted. The levels of benefit to be tested in the trial, if demonstrated, will result in important reductions in mortality, hospital and institutional admissions and improved quality of life. These results will be relevant, not only to the UK older population but also to many other countries and settings where health care of older people has become a policy priority.

\section{Competing interests}

None declared

\section{Authors contributions}

All authors contributed to the design of the trial. AF wrote the manuscript and DJ, CB and AT provided comments. All authors approved the final manuscript.

\section{Acknowledgements}

Sponsor: Medical Research Council, Department of Health, Scottish Office

Trial Steering Committee: Professor Sir John Grimley Evans (Chair from Jan 200I), Professor Carol Brayne, Linda Davies (University of Manchester),
Professor Mike Drummond (University of York), Professor Andy Haines (Chair 1994-2000), Professor Karen Luker, Dr Madge Vickers.

Practices from the Medical Research Council General Practice Research Framework (MRC GPRF): Director Dr Madge Vickers

\section{References}

I. Williamson J, Stokoe IH, Gray S, et al: Old people at home:their unreported needs Lancet 1964, I: I I I7-20

2. Thomas P: Experiences of two preventive clinics for the elderly. $B M J$ I 968, 2:357-360

3. Williams El, Bennett FM, Nixon JV, et al: Socio medical study of patients over 75 in general practice. $B M$ J 1972, 2:445-448

4. Kane RA, Kane RL: Assessing the Elderly. Lexington, Massachussets, Lexington Books I98I

5. Barber $\mathrm{JH}$, Wallis JB: The effects of a system of geriatric screening and assessment on general practice workload Health Bulletin 1982, 40:125-132

6. Taylor R, Ford G, Barber $\mathrm{JH}$ : The elderly at risk. A critical review of problems and progress in screening and case-finding. Age Concern Research Perspective Monograph No 6 Mitcham Surrey: Age Concern

7. Barber $\mathrm{JH}$, Wallis J, McKeating $\mathrm{E}$ : A postal screening questionnaire in preventive geriatric care. J $R$ Coll Gen Pract 1985, 35:288290

8. Bowns I, Challis D, Tong MS: Case finding in elderly people: validation of postal questionnaire $\mathrm{Br} J$ Gen Prac I991, 4 I: I00-104

9. Tulloch AJ, Moore V: A randomised controlled trial of geriatric screening and surveillance in general practice. J Roy Coll Gen Pract 1979, 29:733-742

10. Vetter NJ, Jones DA, Victor CR: Effect of health visitors working with elderly patients in general practice: a randomised controlled trial. BMJ 1984, 288:369-372

II. Hendriksen C, Lund E, Stromgard E: Consequences of assessment and intervention among elderly people: a three-year randomised controlled trial. BM] 1984, 289:1522-4

12. Hendriksen C, Lund E, Stromgard E: Hospitalization of elderly people. A 3-year controlled trial. J Am Geriatr Soc 1989, 37: I I722

13. Department of Health: Terms of service for doctors in general practice. London DOH 1989

14. Royal College of General Practitioners: Preventive care of the elderly: a review of current developments. Occasional Paper 35, Royal College of General Practitioners (Edited by: Taylor RC, Buckley EG) 1987

I5. Rubenstein LZ, Stuck AE, Siu AL, Wieland D: Impacts of geriatric evaluation and management programs on defined outcomes: overview of the evidence. I Am Geriatr Soc 1991, 398S- I6S

16. Royal College of General Practitioners: Health checks for people aged 75 and over. Occasional Paper 59, Royal College of General Practitioners (Edited by: Williams El, Wallace P) 1993

17. Swan IR, Browning GG: The whispered voice as a screening test for hearing impairment. I $R$ Coll Gen Pract 1985, 35:197

18. McGraw PV, Winn B: Glasgow Acuity Cards: a new test for the measurement of letter acuity in children. Ophthal Physiol Opt 1993, 13:400-404

19. Folstein MF, Folstein SE, McHugh PR: "Mini-mental state". A practical method for grading the cognitive state of patients for the clinician. I Psychiatr Res 1975, I 2:189-98

20. Sheik JI, Yesavage JA: Geriatric Depression Scale (GDS): Recent evidence and development of a shorter version. Clinical Gerontology 1986, 37:819-20

21. Bulpitt CJ, Shipley MJ, Demirovic J, Ebi-Kryston KL, Markowe HL, Rose G: Predicting death from coronary heart disease using a questionnaire Int J Epidemiol 1990, I 9(4):899-904

22. Stockley $\mathrm{IH}$ : A pocket aide-memoire on drug interactions $\mathrm{Am}$ Hosp Pharm 1975, 32(4):395-397

23. Bulpitt CJ, Benos A, Nicholl C, Fletcher AE: Should medica screening of the elderly be promoted? Gerontology 1990, 36:230245

24. Bergner M, Bobbit RA, Carter WB, Gilson B: The Sickness Impact Profile: development and final revision of a health status measurement. Med Care 1981, 19:787-805

25. Powell-Lawton M: The Philadelphia Geriatric Centre Morale Scale. A revision. / Gerontology 1975, 30:85-89 
26. Shipley MJ, Smith PG, Dramaix M: Calculation of power for matched pair studies when randomization is by group. Int J Epidemiol 1989, 18(2):457-461

27. Office of Population Censuses and Surveys: Mortality statistics Area Series DH5 No 10 1991

28. Department of Health Office of Population Censuses and surveys: Hospital In-Patient Enquiry Series MB4 no 29

29. Hsieh FY: Sample size formulae for intervention studies with the cluster as unit of randomization. Stat Med 1988, 7(II): II95-120I

30. Fletcher AE: Multidimensional assessment of elderly people. British Medical Bulletin 1998, 54:945-960

31. Stuck AE, Siu AL, Wieland GD, Adams J, Rubensteiun LZ: Comprehensive geriatric assessment: a meta-analysis of controlled trials. Lancet 1993, 342:1032-6

32. van Haastregt JCM, Diederiks JPM, van Rossum E, de Witte LP, Crebolder HFJM: Effects of prevenetive home visists to elderly people living in the community: a systematic review. $B M J$ 2000, 320:754-8

33. Elkan R, Kendrick D, Dewey M, Hewitt M, Robinson J, Blair M, Williams D, Brummell : Effectiveness of home based support for older people: systematic review and meta-analysis. BMJ 200I, 323:719-725

34. Stuck AE, Egger M, Hammer A, Minder CE, Beck JC: Home visits to prevent nursing home admission and functional decline in elderly people: systematic review and meta-regression analysis. JAMA 2002, 27;287(8): 1022-8

35. Department of Health: National service framework for older people. London: $\mathrm{DoH} 200 \mathrm{I}$

\section{Pre-publication history}

The pre-publication history for this paper can be accessed here:

http://www.biomedcentral.com/1472-6963/2/21/prepub 